\title{
Validation of cutoffs for skeletal muscle mass index based on computed tomography analysis against dual energy X-ray absorptiometry in patients with cirrhosis: the KIRRHOS study
}

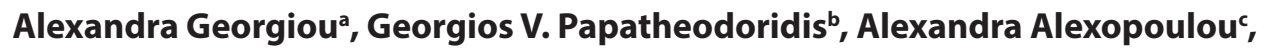 \\ Melanie Deutschc, loannis Vlachogiannakos ${ }^{b}$, Panagiota loannidou $^{b}$, Maria-Vasiliki Papageorgiou ${ }^{b}$, \\ Nikolaos Papadopoulos ${ }^{d}$, Mary Yannakoulia ${ }^{a}$, Meropi D. Kontogiannia \\ Harokopio University, Athens; Medical School of National and Kapodistrian University of Athens, Laiko General \\ Hospital; Medical School of National and Kapodistrian University of Athens, Hippokration General Hospital; Army \\ Share Fund Hospital of Athens, Athens, Greece
}

\section{Abstract}

\begin{abstract}
Background Accurate assessments of muscle mass in patients with cirrhosis are necessary in clinical practice. Computed tomography (CT) of the upper abdomen has been proposed as a useful method for quantifying muscle mass. Recently, Carey et al developed specific cutoffs for muscle wasting based on the skeletal muscle index at the L3 vertebra (L3-SMI) for cirrhotic patients. The aim of the present study was to assess the concurrent validity of the newly proposed cutoffs of Carey et al, along with others widely used in several clinical contexts, using dual energy X-ray absorptiometry (DXA) as the reference method.

Methods Data were evaluated from 97 Caucasian patients (59.8\% male, 59.1 \pm 11.6 years old, $45.4 \%$ decompensated) with cirrhosis of various etiologies. Muscle mass was assessed using the appendicular lean mass index (ALMI) by DXA and the L3-SMI by CT. Low L3-SMI was defined in relation to 5 different cutoffs.

Results Low muscle mass prevalence was $13.4 \%$ according to ALMI and $26.8-45.4 \%$ according to the different cutoffs applied for L3-SMI. The Carey et al, Prado et al and Montano-Loza et al cutoffs showed similar sensitivity (all $69.2 \%$ ) and specificity (79.8\%, 76.2\% and 75.0\%, respectively) and high accuracy (78.4\%, $75.3 \%$ and $74.2 \%)$. The Carey et al cutoffs showed the highest diagnostic validity against DXA: the multivariate odds ratio adjusted for age, sex, body mass index category, disease etiology and model for end-stage liver disease score (95\% confidence interval) was 5.88 (1.36-25.4), $\mathrm{P}=0.018$.
\end{abstract}

Conclusion Compared to DXA, the cutoffs for identifying muscle wasting proposed by Carey et al were proven to be the most accurate.

Keywords Cirrhosis, muscle mass, muscle wasting, DXA, computed tomography

Ann Gastroenterol 2020; 33 (1): 1-7
${ }^{\mathrm{a} D e p a r t m e n t ~ o f ~ N u t r i t i o n ~ a n d ~ D i e t e t i c s, ~ S c h o o l ~ o f ~ H e a l t h ~ S c i e n c e s ~}$ and Education, Harokopio University (Alexandra Georgiou,

Mary Yannakoulia, Meropi D. Kontogianni); ' Department of Gastroenterology, Medical School of National and Kapodistrian University of Athens, Laiko General Hospital of Athens (Georgios V. Papatheodoridis, Ioannis Vlachogiannakos, Panagiota Ioannidou, Maria-Vasiliki Papageorgiou); ${ }^{\text {nd }}$ Department of Internal Medicine, Medical School of National and Kapodistrian University of Athens, Hippokration General Hospital of Athens, Athens, Greece (Alexandra Alexopoulou, Melanie Deutsch); ${ }^{\mathrm{d}} 1^{\text {st }}$ Department of Internal Medicine, Army Share Fund Hospital of Athens (Nikolaos Papadopoulos), Athens, Greece

\section{Conflict of Interest: None}

Correspondence to: Meropi D. Kontogianni, Assistant Professor, Department of Nutrition and Dietetics, School of Health Sciences and Education, Harokopio University, 70 El. Venizelou Ave, 17671, Kallithea, Greece, e-mail: mkont@hua.gr

Received 31 July 2019; accepted 29 October 2019; published online 21 November 2019

DOI: https://doi.org/10.20524/aog.2019.0432

\section{Introduction}

Muscle wasting is quite prevalent in cirrhosis, because of the metabolic changes that occur because of liver malfunction [1]. Lately, muscle mass quantification methods have attracted a lot of attention among the scientific community, mainly because of the significant negative impact of muscle wasting on the manifestation of disease complications and survival in patients with advanced liver disease [2]. Therefore, it has been suggested that muscle mass assessment should be performed routinely in this group of patients, using validated but also widely available techniques.

Radiological methods, i.e., dual energy X-ray absorptiometry (DXA) and computed tomography (CT) image analysis, have been proposed for muscle mass quantification in liver disease patients [3]. DXA, although costly and not widely available in clinical practice, is considered a highly accurate method, since it overcomes the possible accuracy 
burden in cases of overhydration by estimating limb muscle mass $[4,5]$. Thus, it has been used as the reference method for the validation of other muscle mass quantification techniques, such as CT [6,7].

Muscle mass area by CT image analysis in the L3 vertebra has been positively correlated with whole body muscle mass in oncology patients [8] and negatively with survival specifically in patients with advanced liver disease [9]. Since this method was originally developed for muscle mass quantification in oncology patients, its application in cirrhotic patients has until recently been performed arbitrarily, using cutoffs developed for the oncological population $[10,11]$. During the past few years, specific cutoff points have been developed for liver disease patients $[12,13]$. The most recent ones, proposed by Carey et al [13], have been supported by the European Association for the Study of the Liver (EASL) for muscle mass assessment of cirrhotic patients, though with a note that additional validation studies are needed [5]. Similarly, Carey et al [13] urged for validation of their proposed cutoff values in other groups of patients. Therefore, the aim of the present study was to assess the concurrent validity of the newly proposed cutoffs of Carey et al, as well as other cutoff points widely used in several clinical conditions, against DXA as the reference method.

\section{Patients and methods}

\section{Study sample}

Ninety-seven Caucasian adult ( $>18$ years old) cirrhotic patients (58 male; mean age 59.1 \pm 11.6 years) from the KIRRHOS study (study for the evaluation of dietary intake, body composition and performance status in cirrhotic patients) for whom CT scans were available participated in this analysis. Patients' enrollment was performed in 2 outpatient liver clinics (Academic Department of Gastroenterology, Laiko General Hospital of Athens, and 2nd Academic Department of Internal Medicine, Hippokratio General Hospital of Athens) from June 2015 to July 2018. The diagnosis of cirrhosis was based on liver biopsy or a combination of clinical, laboratory and imaging data (Fibroscan values $>14 \mathrm{kPa}$ or clinical picture of decompensated cirrhosis, i.e., manifestation of ascites, edema, esophageal or gastric varices and/or encephalopathy). Exclusion criteria included pregnancy or lactation, presence of hepatocellular or other forms of cancer, hepatic coma, diagnosed acquired immunodeficiency syndrome, renal or pancreatic insufficiency and active enteral feeding. Patients with alcoholic cirrhosis were included in the study only if alcohol intake had already ceased for at least one month before enrollment in the study. A consent form was signed by all participants, after they had been fully informed about the aims of the study. The protocol was approved by the Bioethics Committee of Harokopio University and the Scientific Committees of all participating Hospitals and it has been registered in the ClinicalTrials.gov system (ID NCT03242798).

\section{Medical assessment}

Medical assessment was implemented by the study physician. Disease etiology and any clinical manifestations related to cirrhosis, such as ascites, edema, esophageal or gastric varices and encephalopathy, as well as their severity, were recorded. Patients were classified as compensated or decompensated, based on the absence or presence of the abovementioned clinical complications, respectively. Recent measurements (within a month prior to baseline evaluation) of total bilirubin, serum creatinine and prothrombin time were also collected from the medical records. Based on the biochemical data collected, the model for end-stage liver disease (MELD) score [14] was calculated for the estimation of disease severity.

\section{Anthropometry and body composition}

Body weight (BW) was measured using an analog scale and height using a stadiometer (Seca 711). Dry weight was calculated by subtracting $5 \%$ of the measured BW for mild ascites, $10 \%$ for moderate ascites and $15 \%$ for tense ascites, with an additional 5\% subtracted if bilateral pedal edema was present, as suggested by EASL [5]. Dry body mass index (BMI) was also calculated [15].

Body composition analysis was performed using the DXA method and the appendicular lean mass index (ALMI, $\mathrm{kg} / \mathrm{m}^{2}$ ) was calculated. Low ALMI was defined using the cutoffs of $<7.59 \mathrm{~kg} / \mathrm{m}^{2}$ for men $\leq 65$ years old and $<7.64$ for men $>65$ years old, and $<5.47 \mathrm{~kg} / \mathrm{m}^{2}$ for women $\leq 65$ years old and $<5.78$ for women $>65$ years old, derived from a Mediterranean population [16]. Muscle mass estimation was also performed by computing the muscle mass area at the level of the L3 vertebra (L3-SM), using appropriate software (SliceOmatic V4.3 software, Tomovision, Montreal, PQ). More specifically, an individual section of the CT scan at the L3 vertebra level was isolated and the areas of the psoas, paraspinal and abdominal wall (including rectus abdominis, transverse abdominis, and internal and external oblique) muscles were outlined. Using the Hounsfield unit (HU) range for which muscle mass absorbs X-rays $(-29,+150)$, the cross-sectional area of the above mentioned muscles was semi-automatically quantified, leading to an estimation of the total cross-sectional area of the abdominal skeletal muscles at the L3 level (L3-SM in $\mathrm{cm}^{2}$ ). The estimated muscle mass area was then normalized to height to calculate skeletal muscle index: L3-SMI $\left(\mathrm{cm}^{2} / \mathrm{m}^{2}\right)=\mathrm{L} 3-\mathrm{SM}$ in $\mathrm{cm}^{2} /$ height ${ }^{2}$ in $\mathrm{m}^{2}$. Five different cutoff values were applied in the present study, most of which had been previously applied in cirrhotic patients:

- $<39 \mathrm{~cm}^{2} / \mathrm{m}^{2}$ for women and $<50 \mathrm{~cm}^{2} / \mathrm{m}^{2}$ for men, developed for cirrhotic patients by Carey et al [13]

- $<42 \mathrm{~cm}^{2} / \mathrm{m}^{2}$ for women and $<50 \mathrm{~cm}^{2} / \mathrm{m}^{2}$ for men, developed for cirrhotic patients by Montano-Loza et al [12]

- $<39 \mathrm{~cm}^{2} / \mathrm{m}^{2}$ for women and $<55 \mathrm{~cm}^{2} / \mathrm{m}^{2}$ for men, also developed for the oncological population by Mourtzakis et al [17] and endorsed by the European Society of Clinical Nutrition and Metabolism (ESPEN) [18] 
- $<41 \mathrm{~cm}^{2} / \mathrm{m}^{2}$ for women, irrespective of dry BMI, and $<43 \mathrm{~cm}^{2} / \mathrm{m}^{2}$ for men with dry BMI $<25 \mathrm{~kg} / \mathrm{m}^{2}$ and $<53 \mathrm{~cm}^{2} / \mathrm{m}^{2}$ for men with dry BMI $\geq 25 \mathrm{~kg} / \mathrm{m}^{2}$, also developed for oncological patients by Martin et al [19]

- $<38.5 \mathrm{~cm}^{2} / \mathrm{m}^{2}$ for women and $<52.4 \mathrm{~cm}^{2} / \mathrm{m}^{2}$ for men, developed for the oncological population by Prado et al [20].

\section{Statistical analysis}

We calculated that a total of 94 patients would be required to evaluate 2 -sided differences at a $5 \%$ significance level (i.e., 95\% confidence interval [95\%CI]), based on the following assumptions: i) the prevalence of low muscle mass in a sample of chronic liver disease patients of various disease stages and etiologies is around 13\% [21]; and ii) the annual population size of cirrhotic patients who visit the outpatient clinics participating in the study and are eligible for recruitment is 200 . The achieved statistical power (i.e., 1 minus Type-II error) was $95 \%$.

Categorical variables were represented as absolute numbers and relative frequencies. The normality of the continuous variables was examined using the Shapiro-Wilk test and graphically through Q-Q plots. Normally distributed continuous variables were expressed as mean values \pm standard deviation (SD) and non-normally distributed as median and $1^{\text {st }}-3^{\text {rd }}$ quartiles. The $\chi^{2}$ test was used for comparisons between categorical variables and the agreement between them was examined using Cohen's kappa coefficient (kappa). The independent samples $t$-test was used for comparison of normally distributed continuous variables between patients with low and adequate muscle mass according to DXA, while the Mann-Whitney test was used for non-normally distributed variables.

The correlation between the quantification of muscle mass as estimated by ALMI, by DXA and L3-SMI by CT analysis was assessed using Pearson's correlation coefficient (r). Study participants' z-scores for muscle mass estimated by ALMI and L3-SMI were calculated according to sex, based on the mean of the ALMI and L3-SMI and the corresponding standard deviations from the study sample. Bland-Altman plots [22] were used to evaluate the relative validity of L3-SMI against ALMI, according to sex. The plots showed the difference between each individual's $\mathrm{z}$-scores derived from ALMI and L3-SMI against their means [22]. The bias (mean difference between ALMI z-scores and L3-SMI z-scores in our study sample) and the corresponding limits of agreement ( $\pm 2 \mathrm{SD})$ of the mean difference were calculated.

Sensitivity, specificity, positive and negative predictive values (PPV and NPV), positive and negative likelihood ratios (PLR and NLR) and accuracy were calculated for the various cutoffs against the reference DXA method. Logistic regression analysis was applied to assess the relationship between muscle wasting identification based on DXA and muscle wasting as identified using 5 different cutoffs based on CT analysis, after adjusting for the following confounders: age (years), sex (male/female), disease etiology (viral/alcoholic/non-alcoholic steatohepatitis/ primary biliary cholangitis [PBC]-autoimmune/unknown), BMI category (underweight/normal weight/overweight/ obese), MELD score, and presence of ascites (yes/no).

\section{Results}

Participants' descriptive characteristics in relation to muscle mass category according to ALMI are presented in Table 1. Among the study participants, $74.3 \%$ had high BW. Chronic viral infection (by hepatitis $\mathrm{B}$ or $\mathrm{C}$ virus) was the leading cause of cirrhosis among study participants, accounting for the $53.6 \%$ of disease etiology. Almost half of the study participants had decompensated cirrhosis (45.4\%). Low muscle mass prevalence according to ALMI was estimated at $13.4 \%$ in the total study sample. Moreover, patients with high BW (overweight and obese altogether) were more likely to have adequate muscle mass compared with normal and underweight patients $(\mathrm{P}=0.041)$. It should also be noted that a higher percentage of patients with alcoholic etiology was found in the low muscle mass subgroup compared with the normal muscle mass group ( $46.2 \%$ vs. $20.2 \%, \mathrm{P}=0.041$ ), while the relevant difference observed for patients with $\mathrm{PBC} /$ autoimmune etiology did not appear to be statistically significant $(0.0 \%$ vs. $16.7 \%, \mathrm{P}=0.112)$. Overall, there were 5 patients with $\mathrm{PBC}$ etiology among the study participants, most of them with compensated cirrhosis (80\%) and with increased BW (80\%), but none with low ALMI $(0 \%)$.

\section{Correlation of muscle mass estimation between ALMI and L3-SMI by CT}

Quantification of muscle mass by estimation of ALMI by DXA and L3-SMI by CT were highly correlated in the total sample $(r=0.613, P<0.001)$ and in both sexes $(r=0.392, P=0.002$ for men and $\mathrm{r}=0.641, \mathrm{P}<0.001$ in women). When the $\mathrm{z}$-scores for each patient were calculated based on the mean of the ALMI and the L3-SMI according to sex (ALMI mean \pm SD for men: $8.17 \pm 1.0$ and for women: $6.99 \pm 0.92$, and L3-SMI mean \pm SD for men: $53.1 \pm 6.6$ and for women: $45.2 \pm 9.1$ ), 2 Bland-Altman plots were constructed to compare the 2 methods of muscle mass quantification based on the relative $\mathrm{z}$-scores according to sex (Fig. 1). The bias and the corresponding limits of agreement $( \pm \mathrm{SD})$ of the mean difference were $0.00( \pm 1.10)$ for men (Fig. $1 \mathrm{~A})$ and $0.07( \pm 0.73)$ for women (Fig. 1B). Based on the plots depicted in Fig. 1 A,B, no statistically significant correlations between average and difference were detected in either sex $(\mathrm{P}=0.996$ for men and $\mathrm{P}=0.269$ in women in the regression analyses between the average and the difference of the $\mathrm{z}$-scores between methods in each sex). Consequently, L3-SMI showed acceptable agreement with the reference method of ALMI in both sexes.

\section{Concurrent validity of 5 cutoffs for muscle mass assessment based on CT analysis against ALMI by DXA}

Compared to ALMI categorization (low vs. adequate) of muscle mass, considered the reference for muscle wasting diagnosis, the Carey et al cutoffs, followed by the Prado et al and the Montano-Loza et al cutoffs, were the most accurate 
(accuracy: $78.4 \%, 75.3 \%$ and $74.2 \%$, respectively) (Table 2), estimating muscle wasting prevalence at $26.8 \%, 29.9 \%$ and $30.9 \%$, accordingly. The cutoffs by Mourtzakis et al showed the highest sensitivity (84.6\%), but low specificity (60.7\%), leading to less accurate results.

To support the findings mentioned above, logistic regressions were performed to examine the probability of a patient having low muscle mass according to the reference method of ALMI when categorized as having low muscle mass based on CT analysis using the 5 different cutoffs.
After adjustment for age, sex, BMI category, disease etiology, presence of ascites and MELD score, patients found to have low muscle mass according to Carey et al cutoffs, had a 5.88 times higher probability of having low muscle mass according to ALMI (95\%CI 1.36-25.4, $\mathrm{P}=0.018$ ). Assessment of low muscle mass with either the Prado et al or the Montano-Loza et al cutoffs was also correlated with low muscle mass according to ALMI (odds ratio [OR] 4.24, 95\%CI 1.01-17.8, $\mathrm{P}=0.049$; and OR 5.27, 95\%CI 1.22-22.8, $\mathrm{P}=0.026$, respectively).

Table 1 Descriptive characteristics of the total study sample in relation to muscle mass adequacy, as identified by ALMI derived from DXA

\begin{tabular}{|c|c|c|c|c|}
\hline Characteristics $^{\star}$ & Total sample $(\mathrm{n}=97)$ & $\begin{array}{l}\text { Low muscle mass } \\
\text { (according to ALMI, } \mathrm{n}=13 \text { ) }\end{array}$ & $\begin{array}{c}\text { Adequate muscle mass } \\
\text { (according to ALMI, } \mathrm{n}=84 \text { ) }\end{array}$ & $\mathrm{P}_{\text {-value }}^{\dagger}$ \\
\hline Age (years) & $59.1 \pm 11.6$ & $61.2 \pm 9.72$ & $58.8 \pm 11.8$ & 0.441 \\
\hline $\begin{array}{l}\text { Sex, n (\%) } \\
\text { Male }\end{array}$ & $58(59.8 \%)$ & $11(84.6 \%)$ & $47(56.0 \%)$ & 0.050 \\
\hline Dry BMI $\left(\mathrm{kg} / \mathrm{m}^{2}\right)$ & $28.1 \pm 4.73$ & $24.6 \pm 4.04$ & $28.6 \pm 4.61$ & 0.004 \\
\hline $\begin{array}{l}\text { BMI categories, n (\%) } \\
\text { Underweight } \\
\text { Normal weight } \\
\text { Overweight } \\
\text { Obese }\end{array}$ & $\begin{array}{c}1(1 \%) \\
24(24.7 \%) \\
38(39.2 \%) \\
34(35.1 \%)\end{array}$ & $\begin{array}{c}1(7.7 \%) \\
5(38.5 \%) \\
6(46.1 \%) \\
1(7.7 \%)\end{array}$ & $\begin{array}{c}0(0.0 \%) \\
19(22.6 \%) \\
32(38.1 \%) \\
33(39.3 \%)\end{array}$ & 0.012 \\
\hline $\begin{array}{l}\text { Cause of cirrhosis, n (\%) } \\
\text { Viral (HBV/HCV) } \\
\text { Alcohol } \\
\text { NASH } \\
\text { PBC-autoimmune } \\
\text { Unknown etiology }\end{array}$ & $\begin{aligned} 52 & (53.6 \%) \\
23 & (23.7 \%) \\
7 & (7.2 \%) \\
14 & (14.4 \%) \\
1 & (1.0 \%)\end{aligned}$ & $\begin{array}{c}6(46.2 \%) \\
6(46.2 \%) \\
1(7.7 \%) \\
0(0.0 \%) \\
0(0.0 \%)\end{array}$ & $\begin{array}{c}46(54.8 \%) \\
17(20.2 \%) \\
6(7.1 \%) \\
14(16.7 \%) \\
1(1.2 \%)\end{array}$ & 0.225 \\
\hline $\begin{array}{l}\text { Disease stage, } \mathrm{n}(\%) \\
\text { Decompensated }\end{array}$ & $44(45.4 \%)$ & $8(61.5 \%)$ & $36(42.9 \%)$ & 0.208 \\
\hline MELD score & $9.4(7.5-12.6)$ & $10.5(7.7-12.7)$ & $9.2(7.5-12.6)$ & 0.582 \\
\hline
\end{tabular}

${ }^{*}$ Data are presented as means $\pm \mathrm{SD}$ or frequencies

${ }^{\dagger}$ Probability values as derived by chi-square test or 2-samples t-test for comparisons between patients with low or adequate muscle mass

ALMI, appendicular lean mass index; DXA, dual energy X-ray absorptiometry; BMI, body mass index; CT, computed tomography; HBV, hepatitis B virus;

HCV, hepatitis C virus; MELD, model for end-stage liver disease; NASH, non-alcoholic steatohepatitis; PBC, primary biliary cholangitis

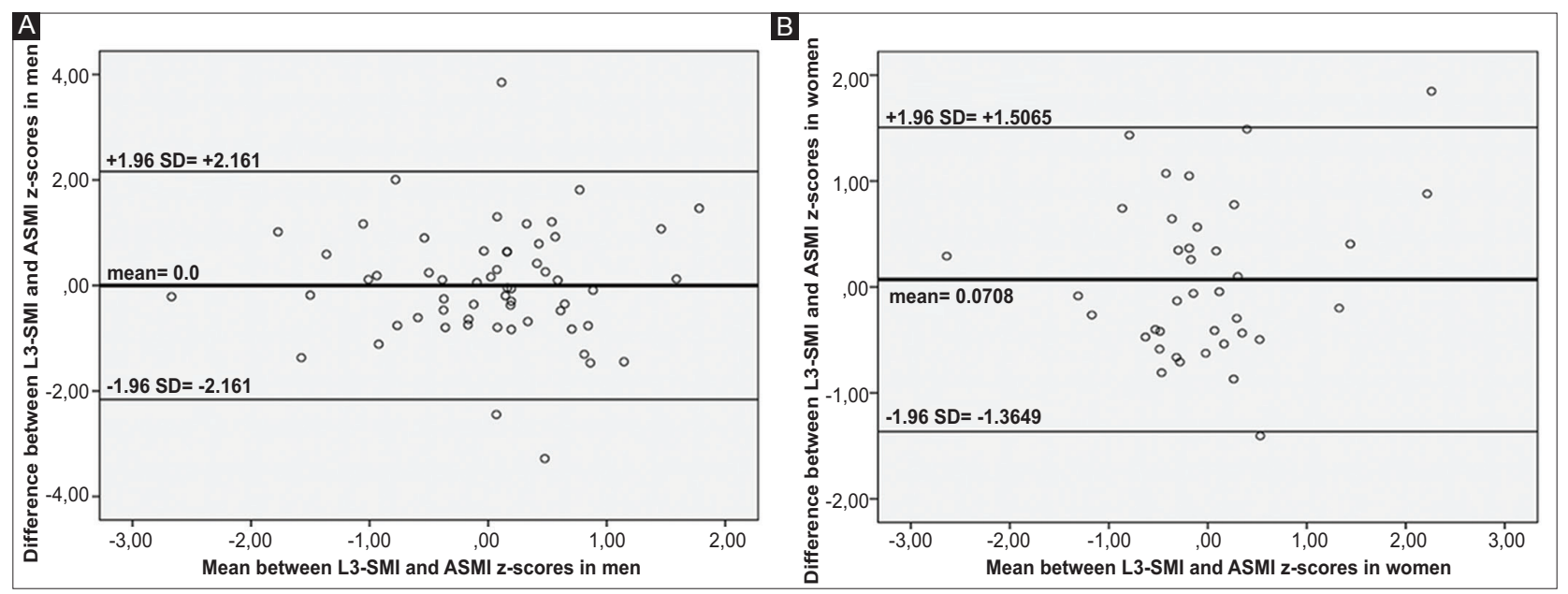

Figure 1 Sex-specific Bland-Altman plots of muscle mass z-scores derived from the ALMI by DXA and the L3-SMI by CT; (A) for men and (B) for women. Represents $95 \%$ limits of agreement between muscle mass z-scores derived from ALMI and L3-SMI

ALMI, appendicular lean mass index; CT, computed tomography; DXA, dual energy X-ray absorptiometry; L3-SMI, skeletal muscle mass index at the L3 vertebra 
Table 2 Validation of 5 different cutoffs for muscle mass assessment based on CT analysis using ALMI by DXA as the reference method in the total sample $(\mathrm{n}=97)$

\begin{tabular}{lcccccccccc}
\hline Cutoffs & Prevalence & Se & Spe & PPV & NPV & PLR & NLR & Accuracy & P-value & kappa \\
\hline Carey et al [13] & $26.8 \%$ & $69.2 \%$ & $79.8 \%$ & $34.6 \%$ & $94.4 \%$ & 3.42 & 0.39 & $78.4 \%$ & $<0.001$ & 0.344 \\
Montano-Loza et al [12] & $30.9 \%$ & $69.2 \%$ & $75.0 \%$ & $30.0 \%$ & $94.0 \%$ & 2.77 & 0.41 & $74.2 \%$ & 0.001 & 0.285 \\
Mourtzakis et al [18] & $45.4 \%$ & $84.6 \%$ & $60.7 \%$ & $25.0 \%$ & $96.2 \%$ & 2.15 & 0.25 & $63.9 \%$ & 0.002 & 0.226 \\
Martin et al [19] & $30.9 \%$ & $53.8 \%$ & $72.6 \%$ & $23.3 \%$ & $91.0 \%$ & 1.97 & 0.64 & $70.1 \%$ & 0.055 & 0.170 \\
Prado et al [20] & $29.9 \%$ & $69.2 \%$ & $76.2 \%$ & $31.0 \%$ & $94.1 \%$ & 2.91 & 0.40 & $75.3 \%$ & 0.001 & 0.299 \\
\hline
\end{tabular}

Low muscle mass prevalence as assessed by the reference method (ALMI by DXA) was $13.4 \%$

*Probability values as derived by Chi-square test for the relation between muscle mass assessment according to the gold standard method DXA and the $\mathrm{CT}$ analysis based on 5 different cutoff points

ALMI, appendicular lean mass index; CT, computed tomography; DXA, dual energy X-ray absorptiometry; NLR, negative likelihood ratio; NPV, negative predictive value; PLR, positive likelihood ratio; PPV, positive predictive value; Se, sensitivity; Spe, specificity

\section{Discussion}

The present study aimed to estimate the validity of the cutoffs newly proposed by Carey et al for L3-SMI assessment by CT in cirrhotic patients, as well as other prominent cutoffs, against the reference method of ALMI. According to our results, the prevalence of low muscle mass in our population sample was estimated to be $11.3 \%$ according to ALMI. When assessed against ALMI, the Carey et al cutoffs showed the best accuracy and agreement compared to the other cutoffs examined, followed by the cutoffs proposed by Prado et al and Montano-Loza et al.

Low muscle mass has a significant prognostic value in liver disease [23]; thus, quantification and assessment of muscle mass in cirrhotic patients can be considered a crucial step in the nutritional assessment and management of these patients, especially in the light of emerging data suggesting possible therapeutic approaches [24]. There are many available methods for the assessment of muscle mass, all showing both strengths and limitations. Anthropometry, such as skinfold thickness measurement, is an easily applicable, rapid and lowcost method; however, it seems to lack accuracy in cirrhotic patients [25]. Bioelectrical impedance has also been applied in patients with advanced liver disease, offering the advantages of a low cost and portable equipment. However, the accuracy of this method is highly affected by the hydration status, unstable in cirrhotic patients [26]. The application of DXA in cirrhotic patients is highly supported [3,5], since the estimation of ALMI, by excluding the muscle mass assessed in the trunk area, overcomes the possible error due to ascites. However, the equipment is quite costly, thus limiting its application to the research area rather than clinical practice [5]. Cross-sectional imaging at the L3 vertebra by CT is widely accepted as a reliable method for direct muscle mass quantification [24], and low muscle mass estimation according to this method has been associated with an increased risk for mortality in cirrhotic patients [23]. The application of CT analysis in cirrhotic patients could be widely supported, as cirrhotic patients frequently undergo CT scanning as part of their medical follow up [5]. The only drawback so far has been the lack of widely accepted and validated cutoffs for this method in patients with advanced liver disease [27]. Thus, muscle mass quantification through CT cross-sectional imaging analysis is emerging as a useful tool in the nutritional assessment of cirrhotic patients, but will find wide acceptance only if specific cutoffs can be established.

In our study, muscle mass quantification by DXA was largely correlated with that by CT analysis. Similar findings were reported by Lindqvist et al in patients with chronic liver disease during the pre-transplant period [28] and by Ishakawa et al in a sample of cirrhotic patients with and without hepatocellular carcinoma [29]. Notably, the prevalence of low muscle mass in our study did not reveal any statistically significant difference in relation to disease etiology in general; however, it should be noted that a significantly higher percentage of patients with an alcoholic etiology was found in the low muscle mass subgroup compared with the normal muscle mass group. This is finding is to be expected considering the detrimental effect of chronic alcohol abuse on muscle mass and protein balance in general [30].

Considering the strong association of the L3-SMI technique with the reference method of ALMI in our study, we sought to examine the validity of the newly proposed cutoffs by Carey et al [13] in comparison with another 4 widely applied cutoffs for L3-SMI assessment. According to our results, the cutoffs by Carey et al, specifically developed for cirrhotic patients, showed the best accuracy, agreement and correlation with the reference method, followed by the cutoffs proposed by Prado et al and Montano-Loza et al, which showed slightly inferior performance. The Carey et al cutoffs, derived from a large multicenter study and endorsed by EASL [5], as well as the Montano-Loza et al cutoffs, have been developed especially for cirrhotic patients; however, they have not yet been widely applied, and only the Carey et al cutoffs have been validated against survival in one study so far [31]. The cutoffs by Prado et al, although non-disease specific, have been previously applied in studies with cirrhotic patients [10,32], but their validity in this group of patients has never been examined before. In our study, the 3 abovementioned cutoffs showed similarly fair agreement, good accuracy and adequate sensitivity and specificity. The NPVs of the cutoffs were high, in contrast to the PPVs, which were quite low (all $<35 \%$ ), indicating that $2 / 3$ of the patients categorized with low muscle mass according to the CT analysis would not be categorized as such by the reference method. 
The present study has both strengths and limitations. To our knowledge, this is the first study ever to comparatively assess the concurrent validity of 5 different cutoffs for muscle mass assessment based on CT analysis in cirrhotic patients. Moreover, the study sample consisted of patients with cirrhosis from various causes, evenly distributed among sex and disease stage, allowing the results to be extrapolated to the whole range of the disease. Regarding limitations, the absence of a gold standard method for muscle mass assessment is a barrier in every study on this topic, creating a risk of bias. Although DXA, applied in the present study, exhibits some drawbacks, such as its high cost and the expertise needed by the operator, it has been suggested by both EASL and ESPEN as a method of muscle mass assessment in liver disease $[3,5]$ and is generally considered a standardized, widely known and valid reference method, since the ALMI value does not appear to be affected by fluid accumulation in cirrhotic patients [4]. Thus, it was chosen in the present study as the reference method for muscle mass assessment.

In conclusion, muscle mass quantification by the reference method DXA showed a large correlation with CT crosssectional imaging analysis. Regarding muscle mass assessment according to L3-SMI, of the 5 different cutoffs implemented in the present study, the Carey et al cutoffs [13] are confirmed to be the most accurate in detecting low muscle mass in cirrhotic patients, closely followed by the ones proposed by Prado et al and Montano-Loza et al. The present study is an additional validation of the diagnostic value of the Carey et al cutoffs and a step towards standardization of these cutoffs as the benchmark for muscle mass assessment in cirrhosis based on CT analysis.

\section{Acknowledgments}

Alexandra Georgiou is co-financed for this research by Greece and the European Union (European Social Fund, ESF) through the Operational Programme "Human Resources Development, Education and Lifelong Learning" in the context of the project "Strengthening Human Resources Research Potential via Doctorate Research" (MIS-5000432), implemented by the State Scholarships Foundation (IKY).

The authors would also like to thank Medella A.E.B.E. for providing access to the CT image analysis software SliceOmatic V4.3 software, Tomovision, Montreal, PQ.

\section{References}

1. Montano-Loza AJ. Muscle wasting: a nutritional criterion to prioritize patients for liver transplantation. Curr Opin Clin Nutr Metab Care 2014;17:219-225.

2. Dasarathy S, Merli M. Sarcopenia from mechanism to diagnosis and treatment in liver disease. J Hepatol 2016;65:1232-1244.

3. Plauth M, Bernal W, Dasarathy S, et al. ESPEN guideline on clinical nutrition in liver disease. Clin Nutr 2019;38:485-521.

4. Belarmino G, Gonzalez MC, Sala P, et al. Diagnosing sarcopenia in male patients with cirrhosis by dual-energy X-Ray absorptiometry estimates of appendicular skeletal muscle mass. JPEN J Parenter

\section{Summary Box}

\section{What is already known:}

- Muscle wasting has a significant negative impact on disease complications and survival in patients with advanced liver disease

- Radiological methods have been proposed for muscle mass quantification in liver disease patients

- Muscle mass area estimation by computed tomography (CT) image analysis has been recognized as a main method for muscle mass quantification globally, but no standard cutoffs have been established

- Recently, Carey et al proposed new cutoffs, which have been endorsed by the European Association for the Study of the Liver but have not yet been validated

\section{What the new findings are:}

- Muscle mass quantification by dual energy X-ray absorptiometry (DXA) is strongly correlated with the results from CT image analysis

- The cutoffs proposed by Carey et al proved to be the most accurate in identifying low muscle mass as assessed by the reference method (i.e., DXA)

Enteral Nutr 2018;42:24-36.

5. European Association for the Study of the Liver. EASL Clinical Practice Guidelines on nutrition in chronic liver disease. J Hepatol 2019;70:172-193.

6. Heymsfield SB. Development of imaging methods to assess adiposity and metabolism. Int J Obes (Lond) 2008;32 (Suppl 7):S76-S82.

7. Mitsiopoulos N, Baumgartner RN, Heymsfield SB, Lyons W, Gallagher D, Ross R. Cadaver validation of skeletal muscle measurement by magnetic resonance imaging and computerized tomography. J Appl Physiol (1985) 1998;85:115-122.

8. Prado CM, Birdsell LA, Baracos VE. The emerging role of computerized tomography in assessing cancer cachexia. Curr Opin Support Palliat Care 2009;3:269-275.

9. van Vugt JL, Levolger S, de Bruin RW, van Rosmalen J, Metselaar HJ, IJzermans JN Systematic review and meta-analysis of the impact of computed tomography-assessed skeletal muscle mass on outcome in patients awaiting or undergoing liver transplantation. $A m \mathrm{~J}$ Transplant 2016;16:2277-2292.

10. DiMartini A, Cruz RJ Jr, Dew MA, et al. Muscle mass predicts outcomes following liver transplantation. Liver Transpl 2013;19:1172-1180.

11. Montano-Loza AJ, Meza-Junco J, Prado CM, et al. Muscle wasting is associated with mortality in patients with cirrhosis. Clin Gastroenterol Hepatol 2012;10:166-173, 173.e1.

12. Montano-Loza AJ, Meza-Junco J, Prado CMM, et al. New cutoff values for sarcopenia for predicting 6-month mortality in cirrhotic patients. J Hepatol 2013;58:S95.

13. Carey EJ, Lai JC, Wang CW, et al; Fitness, Life Enhancement, and Exercise in Liver Transplantation Consortium. A multicenter study to define sarcopenia in patients with end-stage liver disease. Liver Transpl 2017;23:625-633. 
14. Freeman RB Jr, Wiesner RH, Harper A, et al; UNOS/OPTN Liver Disease Severity Score, UNOS/OPTN Liver and Intestine, and UNOS/OPTN Pediatric Transplantation Committees. The new liver allocation system: moving toward evidence-based transplantation policy. Liver Transpl 2002;8:851-858.

15. Garrow JS, Webster J. Quetelet's index (W/H2) as a measure of fatness. Int J Obes 1985;9:147-153.

16. Coin A, Sarti S, Ruggiero E, et al. Prevalence of sarcopenia based on different diagnostic criteria using DEXA and appendicular skeletal muscle mass reference values in an Italian population aged 20 to 80. J Am Med Dir Assoc 2013;14:507-512.

17. Mourtzakis M, Prado CM, Lieffers JR, Reiman T, McCargar LJ, Baracos VE. A practical and precise approach to quantification of body composition in cancer patients using computed tomography images acquired during routine care. Appl Physiol Nutr Metab 2008;33:997-1006.

18. Biolo G, Cederholm T, Muscaritoli M. Muscle contractile and metabolic dysfunction is a common feature of sarcopenia of aging and chronic diseases: from sarcopenic obesity to cachexia. Clin Nutr 2014;33:737-748.

19. Martin L, Birdsell L, Macdonald N, et al. Cancer cachexia in the age of obesity: skeletal muscle depletion is a powerful prognostic factor, independent of body mass index. J Clin Oncol 2013;31:1539-1547.

20. Prado CM, Lieffers JR, McCargar LJ, et al. Prevalence and clinical implications of sarcopenic obesity in patients with solid tumours of the respiratory and gastrointestinal tracts: a population-based study. Lancet Oncol 2008;9:629-635.

21. Hayashi M, Abe K, Fujita M, Okai K, Takahashi A, Ohira H. Association between sarcopenia and osteoporosis in chronic liver disease. Hepatol Res 2018;48:893-904.

22. Bland JM, Altman DG. Statistical methods for assessing agreement between two methods of clinical measurement. Lancet 1986;1:307-310.

23. Kim G, Kang SH, Kim MY, Baik SK. Prognostic value of sarcopenia in patients with liver cirrhosis: A systematic review and metaanalysis. PLoS One 2017;12:e0186990.

24. Montano-Loza AJ. Clinical relevance of sarcopenia in patients with cirrhosis. World J Gastroenterol 2014;20:8061-8071.

25. Fiore P, Merli M, Andreoli A, et al. A comparison of skinfold anthropometry and dual-energy X-ray absorptiometry for the evaluation of body fat in cirrhotic patients. Clin Nutr 1999;18:349-351.

26. Morgan MY, Madden AM, Jennings G, Elia M, Fuller NJ. Twocomponent models are of limited value for the assessment of body composition in patients with cirrhosis. Am J Clin Nutr 2006;84:1151-1162.

27. Tandon P, Raman M, Mourtzakis M, Merli M. A practical approach to nutritional screening and assessment in cirrhosis. Hepatology 2017;65:1044-1057.

28. Lindqvist C, Brismar TB, Majeed A, Wahlin S. Assessment of muscle mass depletion in chronic liver disease: Dual-energy x-ray absorptiometry compared with computed tomography. Nutrition 2019;61:93-98.

29. Ishikawa T, Hoshii A, Hokari T, et al. Correlation between dualenergy X-ray absorptiometry (DXA) method and computed tomography (CT) measurement method for diagnosis of sarcopenia in chronic liver disease. Int J Dev Res 2018;8:18307-18309.

30. Dasarathy J, McCullough AJ, Dasarathy S. Sarcopenia in alcoholic liver disease: clinical and molecular advances. Alcohol Clin Exp Res 2017;41:1419-1431.

31. Kang SH, Jeong WK, Baik SK, Cha SH, Kim MY. Impact of sarcopenia on prognostic value of cirrhosis: going beyond the hepatic venous pressure gradient and MELD score. J Cachexia Sarcopenia Muscle 2018;9:860-870.

32. Cruz RJ Jr, Dew MA, Myaskovsky L, et al. Objective radiologic assessment of body composition in patients with end-stage liver disease: going beyond the BMI. Transplantation 2013;95:617-622. 